\title{
Right but not left angular gyrus modulates the metric component of the mental body representation: a tDCS study
}

\author{
Grazia Fernanda Spitoni · Giorgio Pireddu • \\ Rocco Luca Cimmino • Gaspare Galati • Alberto Priori • \\ Michal Lavidor $\cdot$ Liron Jacobson $\cdot$ Luigi Pizzamiglio
}

Received: 18 February 2013 / Accepted: 21 April 2013 / Published online: 12 May 2013

(c) The Author(s) 2013. This article is published with open access at Springerlink.com

\begin{abstract}
The parietal lobes contribute to body-space representation. The present work aims at characterizing the functional role of the inferior parietal lobe in bodyspace representation and at studying the different roles of the angular gyrus in the right and left hemisphere. We conducted three separate transcranial direct current stimulation (tDCS) experiments using "tactile distance task" as an implicit measure of body representation. Whereas anodal tDCS on the right angular gyrus influences vocal reaction times (vRT) for stimuli delivered on the ipsilateral body parts without changes of accuracy, right tDCS improved both vRT and accuracy for tactile stimuli on the contralateral limbs. Sham or left parietal anodal tDCS had no effect. These evidences support the view that right parietal areas
\end{abstract}

G. F. Spitoni $(\bowtie) \cdot$ G. Pireddu $\cdot$ R. L. Cimmino $\cdot$ G. Galati L. Pizzamiglio

Department of Psychology, The Sapienza University of Rome, Rome, Italy

e-mail: grazia.spitoni@uniroma1.it

G. F. Spitoni · G. Pireddu · R. L. Cimmino · G. Galati ·

L. Pizzamiglio

Neuropsychology Laboratory, IRCCS Santa Lucia Foundation, Rome, Italy

\author{
A. Priori \\ Dipartimento di Scienze Neurologiche, Università degli Studi \\ di Milano, Fondazione IRCCS Ospedale Maggiore Policlinico, \\ Milan, Italy \\ M. Lavidor · L. Jacobson \\ The Leslie and Susan Gonda Multidisciplinary Brain Research \\ Center, Bar-Ilan University, Ramat Gan, Israel
}

M. Lavidor · L. Jacobson

Department of Psychology, Bar-Ilan University,

Ramat Gan, Israel have a crucial role in the metric component of the body representation.

Keywords Body representation · Touch · tDCS · Angular gyrus

\section{Introduction}

We are constantly requested to process information in the environment regarding the position of all objects surrounding our body and our body itself. A theoretical framework that well describe the relations between the sense of touch and the body has been proposed by Serino and Haggard (2010). The authors describe an analytical model in which tactile afferent information can either be influenced by, or have influence upon a mental body representations (MBRs). Following the authors' model, four separate processes are necessary to link the physical body, the sense of touch, and the mental representation of one's own body: (1) The physical body organizes tactile sensations, (2) Tactile sensations contribute to a mental MBR, (3) MBRs reciprocally influence primary tactile processing, and (4) MBRs mediate the formation of object representation from primary tactile sensations.

This latter process seems particularly important whenever one is asked to consider the intrinsic properties of a given object touching the skin. For example, it has been shown that altering the visual experience of the body alters perceived tactile distances (Cardini et al. 2012; Longo et al. 2008; Taylor-Clarke et al. 2004): This evidence is interpreted as the brain attempt to preserve tactile size constancy by rescaling the primary, distorted body-surface representation into object-centered space according to visual experience of the body. A further study by de Vignemont 
et al. (2005) developed this issue showing that proprioception, as well as vision, plays a role in the formation of a MBR mediating tactile distance judgement. The authors also showed that the MBR used for body referencing of touch is not only a memorized body template stored in long-term memory, but also is updated online to integrate current sensory information. From this perspective, Serino and Haggard (2010) claimed that tactile perceptions are always referenced to the body, even if the content of the perception is an external object.

The right parietal lobe contributes to body representation in several ways. Tsakiris et al. (2008) used TMS to show that the right temporal parietal junction (rTPJ) supports an internal model of the body. They suggested that the internal model of the body acts as a stored template against which novel stimuli are compared, allowing the maintenance of a basic sense of embodied self. Various functional magnetic resonance imaging (fMRI) studies investigated the properties of the parietal lobe in processing body information (see Corradi-Dell'Acqua et al. 2008; Pellijeff et al. 2006). Parkinson et al. (2010) showed that the superior parietal lobule (SPL) maintains an up-to-date estimate of the current postural configuration of the part of the body that is used during the planning and the execution of a reaching movement. Dijkerman and de Haan (2007) described a model that specifies separable functional entities for somatosensory processing subserving perception and action. The authors propose that action-related processing occurs mainly in the posterior parietal cortex (PPC), whereas recognition and perception involve the insula, as well as the PPC. Relevant to our issue, this model also distinguishes between somatosensory processing about the body (where you have been touched) and about external stimuli (e.g., surface features of objects). On the basis of neurophysiological and human lesions studies, Dijkerman and de Haan suggested a key role of PPC in the metric aspects of the body, such as its spatial configuration and size, whereas the insula is more concerned with higher order somatosensory processing of the body that is either related to a sense of ownership or to emotional experience.

The importance of the parietal lobe in dealing with the localization of somatic stimuli on the skin was previously highlighted by Van Boven et al. (2005). Authors described two areas selectively involved in a grating orientation task (stimulus orientation) and in a simple tactile localization task (stimulus location). In contrast to grating orientation which specifically activates IPL, tactile localization preferentially engages the TPJ.

In this study, we will focus on the metric component of the MBR.

Specifically, we refer to the concept of metric component of body representation as one of the functions that the somatoperception uses to form a model of body size and shape (see Longo et al. 2010). Moreover, in a previous work (Spitoni et al. 2010), we argued that the right angular gyrus may house the process of body referencing of tactile inputs that is required to compare the tactile distance across different body part. Among this frame of evidences, we used the term metric component of body representation to describe a selective component of the body representation used to process tactile distances discrimination.

Despite a rich description of the functional properties of the parietal areas in dealing with body representations, little is known about the neural basis of the specific metric component of body representation. In a previous work (Spitoni et al. 2010), we pointed out the importance of parietal structures in dealing with the perception of the metric component of MBR. In that study, subjects were stimulated with pairs of wooden cylinders separated by a variable distance and attached to nylon filaments of variable length and had to evaluate either the distance between the two cylinders (distance task) or the amount of skin deformation generated by the touch (contact task). The distance but not the contact task implicitly required to access a stored metric representation of the touched body part. Results showed that, while both tasks bilaterally activated parietal and frontal areas, only the evaluation of distances on the body surface activated the angular gyrus and the temporo-parieto-occipital junction in the right hemisphere. Our conclusion was that the right angular gyrus, together with other cortical sensory modalities such as proprioception, plays a key role in the metric tactile perception by linking touched locations on the skin to an internal representation of body parts.

The specific role of the inferior and posterior part of the parietal lobe and the asymmetry between the left and right areas in controlling the metric component of body perception have not been reported by other studies; it is important to verify this finding using a different technique. Transcranial direct current stimulation (tDCS) allows a direct comparison of the effect of the stimulating areas of interest, while the subjects are performing a tactile distance task on the skin of their body.

We delivered tactile stimulations to the left and right limbs and hypothesized that anodal stimulation of the crucial right parietal areas would improve behavioral responses in the same metric distance task that we used in the fMRI study previously described (Spitoni et al. 2010), while anodal stimulation of the same parietal regions in left hemisphere would not enhance subjects' performance in the same way.

Three separate and consecutive experiments were planned. The first aimed at testing the effectiveness of tDCS on a tactile distance discrimination task. It has been known from the previous literature that anodal tDCS applied to the primary somatosensory cortex (S1) improves performance in tactile discriminative tasks only in the parts of the body 
contralateral to the site of stimulation (Ragert et al. 2008). To our knowledge, no data are available on the actual efficacy of tDCS on higher order tactile tasks such as distance/ metric discrimination tasks. The second study tested the lateralization hypothesis, that is, the effect of anodal tDCS on the right versus the left angular gyrus. The third dealt with the effect of anodal tDCS on the right side tested over the ipsilateral versus the contralateral arms.

Since several studies agreed on the facilitative effect of anodal tDCS on cognitive tasks but not on the regular suppressive effect of cathodal stimulation (see Vallar and Bolognini 2011), we decided to focus on anodal stimulation.

Finally, we investigated the possible change of the effects in the time window of stimulations. To this aim, different epochs of stimulations session of Experiments 2 and 3 were considered.

\section{Experiment 1}

The experimental paradigm was an adaptation of the fMRI study by Spitoni et al. (2010). Two pairs of tactile stimuli were rapidly delivered in close succession on two distinct body locations. Subjects concentrated on the body distance subtended by the two items in each pair and judged in which pair the distance was longer.

We used a mixed design with stimulation type (anodal or sham) as a between-subject factor, and time (before or after stimulation) and difficulty (easy or difficult) as within-subject factors.

\section{Methods}

\section{Participants}

There were 13 subjects ( 8 males) in the anodal group (mean age 26.2, mean handedness score 95), and 9 subjects (4 males) in the sham group (mean age 27, mean handedness 95-Edinburgh Inventory, Oldfield 1971; Salmaso and Longoni 1985). The experiment was approved by the Research Ethics Committee of Bar-Ilan University.

\section{Stimuli and task}

The protocol was similar to that used in fMRI study by Spitoni et al. (2010). Stimuli were two sets of 5 perspex inverted $U$-shaped (see Fig. 1) objects, in which the distance between the two touching ends varied from 5 to $9 \mathrm{~cm}$. In each trial, participants were manually stimulated first on the left forearm and $1 \mathrm{~s}$ later on the left thigh and judged which tactile stimulus was longer (distance task). Either the first or the second stimulus was a standard stimulus (distance $=7 \mathrm{~cm}$ ), while the

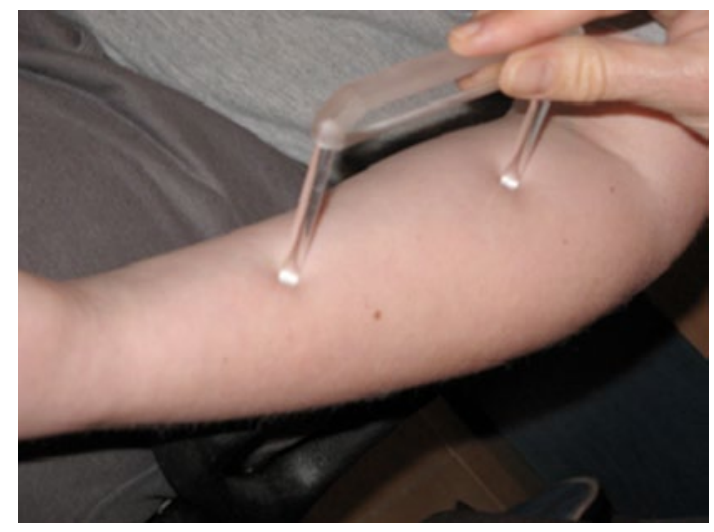

Fig. 1 Stimulus used in the tactile distance task for Experiment 1 (manual stimulation)

other one was either longer or shorter. There were 48 trials in total, in a pseudo-randomized and pre-ordered sequence, balanced for the location of the standard stimulus (forearm or thigh), the location of the longer stimulus (forearm or thigh), and difficulty (easy or difficult). Easy trials were those where the two stimuli differed by $2 \mathrm{~cm}$ (i.e., the nonstandard stimulus was 5 or $9 \mathrm{~cm}$ long); difficult trials were those where the two stimuli differed by $1 \mathrm{~cm}$ (i.e., the nonstandard stimulus was 6 or $8 \mathrm{~cm}$ long). Subjects were instructed to respond vocally, by saying "arm" or "thigh" aloud, as fast and as accurately as possible. Vocal reaction times (vRTs) and accuracy were recorded by a custom made script.

\section{tDCS protocol}

A direct current of $1 \mathrm{~mA}$ (10 min) was induced by two saline-soaked surface sponge electrodes $(7 \times 4.5 \mathrm{~cm})$ and delivered by a battery-driven, constant-current stimulator (Rolf Schneider Electronics, Germany). Previous studies have reported that this stimulation intensity is safe to use in healthy volunteers (Brunoni et al. 2012). In the stimulation sessions, current increased in ramp-like fashion from 0 to $1 \mathrm{~mA}$ at the onset of stimulation, eliciting a transient tingling sensation on the scalp (Hummel et al. 2005). Fifteen minutes after the onset of tDCS anodal or sham sessions the current was turned off slowly over a few seconds. In the sham group, the current turned off after $30 \mathrm{~s}$.

Each participant performed the distance task once (prestimulation); then, he was stimulated over the right angular gyrus in one of the two stimulation conditions (anodal or sham) and then performed the distance task again (poststimulation). The anodal electrode was placed over the right angular gyrus and the cathodal electrode over the left orbitofrontal cortex. Localization was established using the 10-20 EEG technique, and the right angular gyrus was marked over P4 (Fuggetta et al. 2006). 
Results

Accuracy and vRTs are shown in Fig. 2a, b. A mixeddesign analysis of variance (ANOVA) was conducted, with stimulation type (anodal and sham) as the between-subject factor and time (pre-stimulation, post-stimulation) and difficulty (short or long distance) as the within-subjects factors. Bonferroni post hoc tests corrected for multiple comparisons $(p<0.05)$ were conducted.

\section{Accuracy}

A significant main effect of difficulty emerged $[F(1$, $29)=23.3 ; p<0.001]$, with more accurate responses $(77.6 \%)$ to easy stimuli than difficult ones $(63.8 \%)$, in agreement with the well-documented distance effect in magnitude processing (Moyer and Bayer 1976). A significant stimulation type by time interaction also emerged $[F(2,29)=4.69 ; p<0.05]$. Accuracy increased following stimulation in the anodal group (from 70.3 to $77.6 \%$ ), but not in the sham group (pre $68.8 \%$, post $68.0 \%$ ). As significant facilitative effects were found for both difficult and easy comparisons, there was no interaction (Fig. 2a).

$v R T s$

A significant main effect of difficulty emerged $[F(1$, $29)=21.3 ; p<0.001]$, with faster responses $(819 \mathrm{~ms})$ to

a

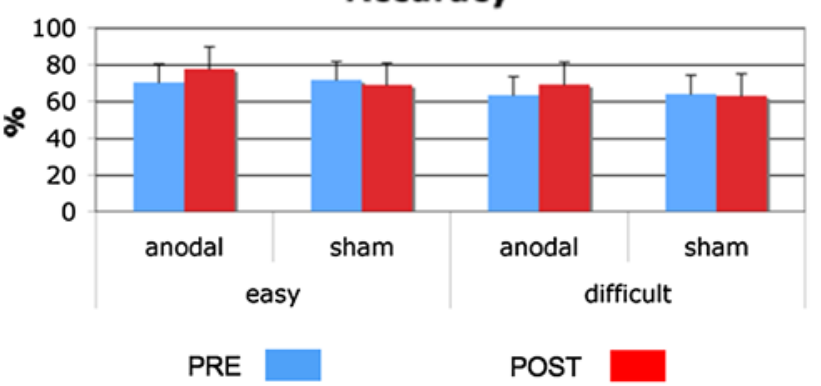

b

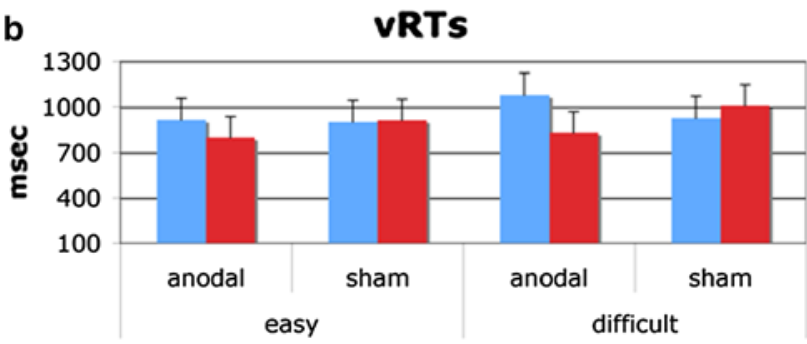

Fig. 2 Results of Experiment 1 (right tDCS stimulation, tactile stimuli on the left arm). a Accuracy (percentage of correct responses). b Vocal response times. Error bars represent standard deviations easy than difficult judgments $(891 \mathrm{~ms})$. A significant effect of time also emerged $[F(1,29)=9.94 ; p<0.01]$, with a reduction of vRTs after stimulation (from 914 to $796 \mathrm{~ms}$ ). Importantly, a significant stimulation type by time interaction emerged $[F(2,29)=3.32 ; p<0.05]$. Following stimulation, vRT decreased in the anodal group (from 1,004 to $821 \mathrm{~ms}$ ), whereas in the sham group, vRTs slightly increased between the two experimental sessions (from 833 to $857 \mathrm{~ms}$ ). The 3-way interaction was not significant (Fig. 2b).

\section{Discussion}

Experiment 1 employed anodal and sham stimulation of the right angular gyrus, while subjects performed the tactile distance task with stimuli delivered on left limbs. The results showed that anodal stimulation improved responses and speed, whereas sham was ineffective. The aim of this first study was to test the effectiveness of tDCS on higher order tactile tasks. As far these preliminary results were in line to our expectations, we carried on Experiments 2 and 3.

\section{Experiment 2}

The second experiment tested the lateralization hypothesis, that is, the effect of anodal tDCS on the right versus the left angular gyrus. This study is strongly linked to that conducted by Spitoni et al. (2010), who found activation in the right angular gyrus after stimulation of the right hemibody; from this standpoint, we aimed at testing the fMRI results not only in the right but also in the left hemisphere.

We used a completely within-subjects design with hemisphere (left or right), stimulation type (anodal or sham), time (before or during stimulation), and difficulty (easy or difficult) as within-subject factors.

All subjects received tactile stimuli in the same "hemibody" of the tDCS stimulation, that is, that if the tDCS was given in the right hemisphere, the tactile stimuli were given to right forearm and to the right thigh. Similarly, when the tDCS was given in the left hemisphere, the tactile stimuli were given to left forearm and to the left thigh.

We predicted that anodal stimulation on the right, but not on the left, angular gyrus would improve performances on the metric distance task.

Methods

\section{Participants}

A total of 12 healthy volunteers ( 6 females) participated in this experiment. All were right-handed, as assessed by a modified version of the Edinburgh Inventory (Oldfield 1971; Salmaso and Longoni 1985). None had a history of 
neurological or psychiatric diseases or were taking medication; all were in good health. Participants' ages ranged from 21 to 34 years (mean age 24.2 ; $\mathrm{SD}=2.9$ ). Each participant was tested in four sessions (with a three-day interval between sessions). All subjects provided written informed consent. The experimental protocol was approved by the Research Ethics Committee of the IRCCS Santa Lucia Foundation of Rome.

\section{Stimuli and task}

We applied tactile stimuli to the forearm and thigh. Test and reference stimuli consisted of two simultaneous contacts from a line of four miniature solenoids. The active solenoids were selected randomly in each trial. Thus, participants experienced tactile distances of one (difficult) or two (easy) steps. We asked them to judge which of the two tactile distances felt longer (arm or thigh).

In $80 \%$ of the trials, the tactile distance on the arm differed from that on the thigh by one or two steps; in the remaining $20 \%$, there was no difference. Before administering the experiment, we calculated the two-point discrimination threshold (defined as the level at which $75 \%$ of the responses were correct) of each participant. In order to make the tactile stimulation above the subjective threshold, we added $0.5 \mathrm{~cm}$ to the two-point discrimination threshold and then used this measure to place the closest pair of solenoids.

Before the experiment, subjects were familiarized with the task in a warm-up session consisting of 10 comparisons.

\section{tDCS protocol}

In the first two sessions, anodal electrode was placed over the right angular gyrus (P4 in the 10-20 EEG system) and the cathode over the right shoulder. In the last two sessions, anodal electrode was place on the left angular gyrus (P3) and cathode still on the right shoulder. Experiment details are reported in Table 1.

Table 1 tDCS protocol of Experiment 2

\begin{tabular}{lllll}
\hline & Pretest & Stimulation & Electrode & Limb \\
\hline I session & Task & Sham & Right AG & Right \\
& $15 \mathrm{~min}$ & & & \\
II session & Task & Anodal & Right AG & Right \\
& $15 \mathrm{~min}$ & & & \\
III session & Task & Sham & Left AG & Left \\
& $15 \mathrm{~min}$ & & & \\
IV session & Task & Anodal & Left AG & Left \\
& $15 \mathrm{~min}$ & & & \\
\hline
\end{tabular}

$A G$ Angular gyrus
In order to balance the administration across participants, half of them started with anodal tDCS and the remaining half with sham.

\section{Results}

We conducted repeated-measures ANOVAs with hemispheres (left and right), stimulation type (anodal or sham), time (before or during stimulation), and difficulty (easy or difficult) as within-subject factors.

A repeated-measures ANOVA on vRTs revealed an highly significant difference among stimulated hemispheres $[F(1,103)=11.740 ; p<0.001]$ with a right-lateralized hemispheric preference. The same repeated-measure ANOVA on accuracy showed that hemispheres did not affect subjects performances. Bonferroni post hoc tests corrected for multiple comparisons $(p<0.05)$ were conducted.

\section{Anodal stimulation of right parietal area and tactile stimuli on right limbs}

Accuracy Here, we found a significant effect for difficulty, with subjects more accurate in judging easier stimuli $(79.4 \%)$ then more difficult ones $(72.5 \%)(F=21.5$; $p<0.001)$.

$v R T s \quad$ Repeated-measure ANOVA showed a significant stimulation type by time interaction $[F(1,103)=17.6 ; p<0.001]$, with faster vRTs during anodal than sham stimulation. Moreover, a significant effect of difficulty emerged $[F(1,103)=48.4$; $p<0.001$ ], with faster responses to easy stimuli. Importantly, a significant stimulation by difficulty interaction emerged $[F(1$, $103)=4.21 ; p<0.05]$, with faster vRTs in the anodal-easy (633 ms) than the anodal-difficult stimulation $(791 \mathrm{~ms})$.

Figure $3 \mathrm{a}, \mathrm{b}$ shows the accuracy and vRTs results after stimulation of the right hemisphere.

\section{Anodal stimulation on left parietal area and tactile stimuli on left limbs}

Again, we performed a 3-way repeated-measures ANOVA with stimulation condition (anodal or sham), time (prestimulation or during stimulation), and difficulty (easy or difficult) as within factors.

Accuracy Also in this case, repeated-measures ANOVA showed that subjects were more accurate in judging easier $(77.7 \%)$ than difficult stimuli $(72.1 \%)[F(1,172)=7.7$; $p<0.01]$.

$v R T$ We found a significant effect for difficulty $[F(1$, $174)=6.4 ; p<0.01]$, with faster vRTs in judging easier $(619 \mathrm{~ms})$ stimuli than more difficult ones (722 ms). 


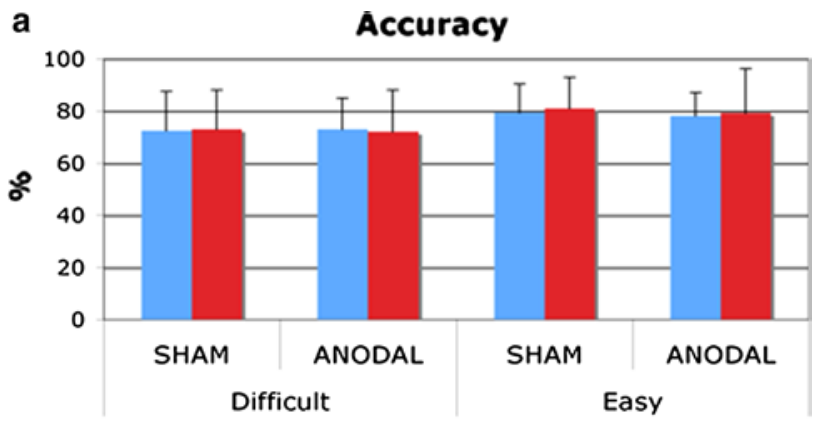

PRE

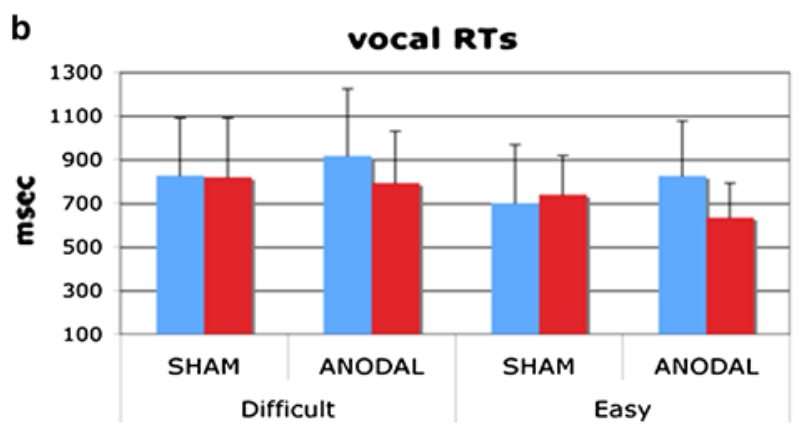

Fig. 3 a, b Experiment 2: accuracy and vocal reaction times when anodal stimulation is given on the RH and the tactile stimuli on the right limbs

Figure 4a, b shows accuracy and vRTs after stimulation of the left hemisphere.

\section{Discussion}

In Experiment 2, anodal and sham stimulation were given on the right and left angular gyrus, while subjects performed the tactile distance task with stimuli delivered on the same side of DC stimulation. Results showed that anodal on right AG significantly improved vRTs and leave unaffected accuracy. No significant effect of anodal stimulation was found when the stimulation was given in the left AG.

\section{Experiment 3}

Experiment 3 dealt with the possible differences in performances when tDCS is given to contra or ipsilateral body parts in respect to the brain stimulation.

We conducted repeated-measures ANOVAs with tactile stimulus side (ipsi-contra), stimulation type (anodal or sham), and difficulty (easy or difficult) as factors.

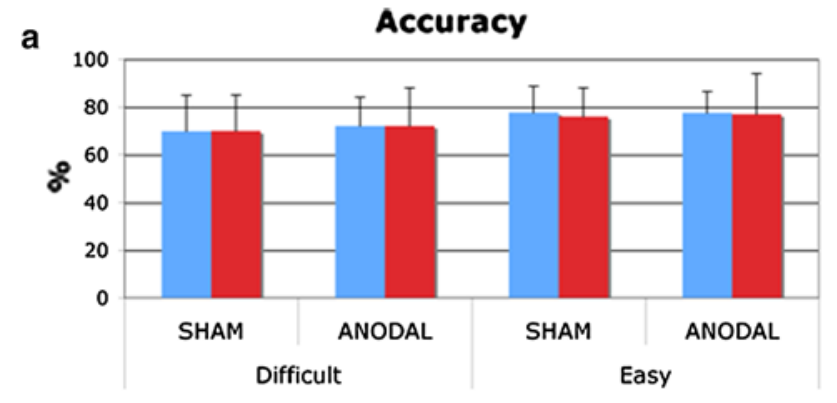

PRE

DURING

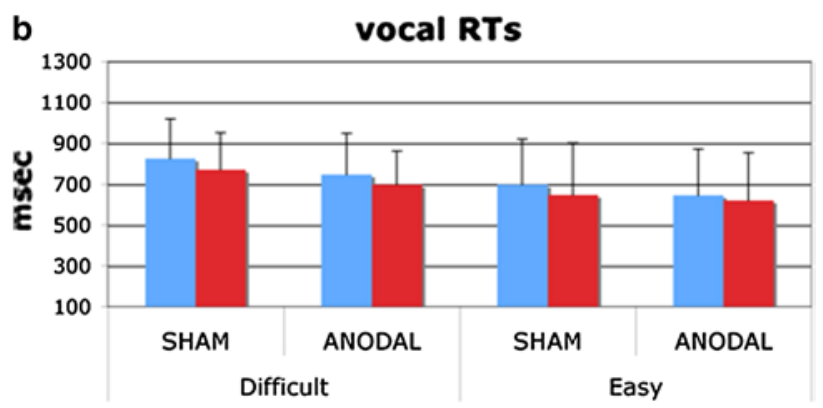

Fig. 4 a, b Experiment 2: accuracy and vocal reaction times when anodal stimulation is given on the $\mathrm{LH}$ and the tactile stimuli on the left limbs

Methods

Participants

In this experiment, we tested 6 participants (3 males) ranged from 19 to 27 (mean age 23.7, $\mathrm{SD}=2.1$ ). Each participant was tested in four sessions (with 2-3 days between each session).

\section{Stimuli and task}

We used the same set of stimuli and the same apparatus of Experiment 2. Experiment 3 details are reported in Table 2.

The order of administration was balanced across subjects.

\section{tDCS protocol}

As shown in Table 2, the tDCS protocol was quite similar to that used in Experiment 2, but in this case, anodal tDCS was always given over the right angular gyrus, and subjects received the tactile stimuli on both contralateral and ipsilateral body parts. Also, in this experiment, we contrasted sham versus anodal stimulation without a pre-stimulation session. 
Table 2 tDCS protocol of Experiment 3

\begin{tabular}{lllll}
\hline & Pretest & Stimulation & Electrode & Limb \\
\hline I session & Task & Sham & Right AG & Left \\
& 15 min & & & \\
II session & Task & Anodal & Right AG & Left \\
& 15 min & & & \\
III session & Task & Sham & Right AG & Right \\
& 15 min & & & \\
IV session & Task & Anodal & Right AG & Right \\
& 15 min & & & \\
\hline
\end{tabular}

$A G$ Angular gyrus

Bonferroni post hoc tests corrected for multiple comparisons $(p<0.05)$ were conducted.

Results

\section{Accuracy}

A main effect of difficulty emerged $[F(1,9)=22.9$; $\left.p<0.001 ; \eta_{\mathrm{p}}^{2}=0.763\right]$, with more accurate responses to easy $(79.1 \%)$ stimuli than difficult ones $(70.7 \%)$.

Moreover, a significant stimulus side by stimulation type by difficulty interaction $[F(1,9)=7.55 ; p<0.05$; $\left.\eta_{\mathrm{p}}^{2}=0.404\right]$ emerged: during anodal stimulation and tactile stimuli on the left limbs accuracy increased in the difficult comparisons (from 71.4 to $79.5 \%$ ), but did not change in the easy comparisons (81.4 and $80.9 \%$ ) (Fig. 5a).

$v R T s$

A significant stimulus side by stimulation type interaction was found with faster vRTs after anodal stimulation and tactile stimuli on the left limb $[F(1,31)=12.6 ; p<0.001$; $\left.\eta_{\mathrm{p}}^{2}=0.63\right]$.

We also found a significant stimulus side by stimulation type by difficulty interaction $[F(1,31)=8.04 ; p<0.05$; $\left.\eta_{\mathrm{p}}^{2}=0.491\right]$, with faster vRTs in difficult comparisons after anodal stimulation when stimuli were administered on right limbs (Fig. 5b).

Figure $5 \mathrm{a}, \mathrm{b}$ shows the accuracy and vRTs results after stimulation of the right hemisphere and tactile stimuli on left or right limbs.

A systematic summary of the main features of the three experiments is given in Table 3.

\section{Discussion}

In the third experiment, anodal and sham stimulation were given only on the right angular gyrus, while subjects performed the tactile distance task with stimuli delivered first a

ACCURACY
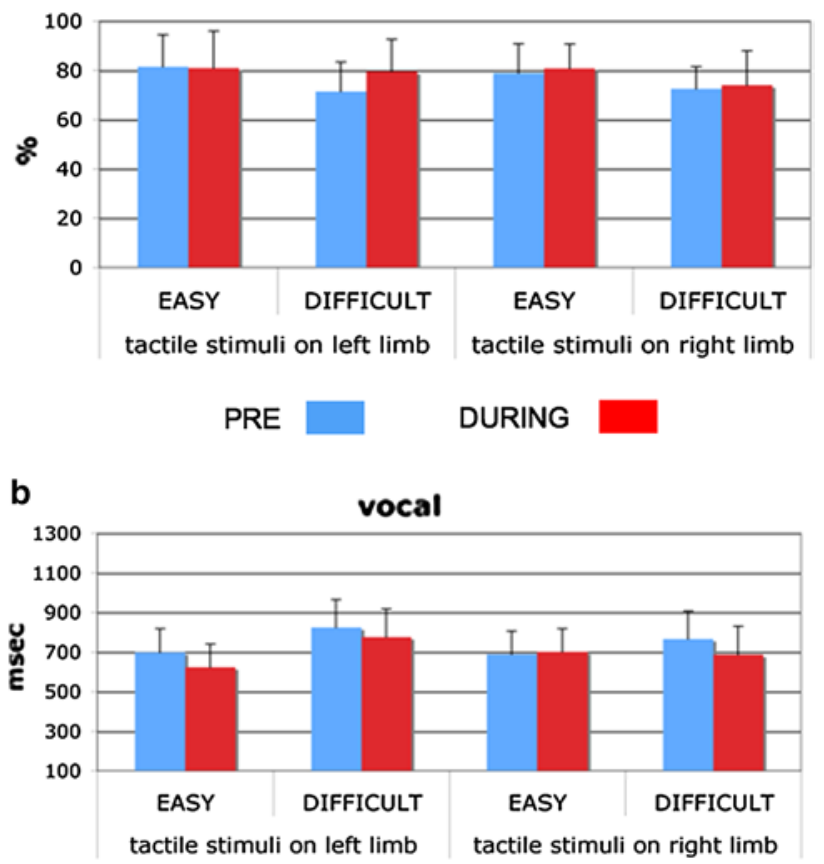

Fig. 5 a, b Accuracy and vocal reaction times when anodal stimulation is given on the RH and the tactile stimuli on the left or the right limbs

on the contralateral and to the ipsilateral hemibody. Here, we found that in case of anodal stimulation on the right $A G$ and tactile task on the left limb, accuracy improved in difficult comparisons. Moreover, vRTs decreased during anodal stimulation.

Also, in the case of anodal on the right AG and tactile task on the right limb, we observed a reduction of vRTs consistent with results of Experiment 2.

A limitation of this third study is the employment of a small sample. For this reason, we believe that the conclusion inferred from the study should be confirmed by further investigations.

\section{Effect of anodal stimulation on the time window of $15 \mathrm{~min}$}

In several studies, the duration of the effects of tDCS spreads from the time period of the stimulation up to about 60 min (Dieckhöfer et al. 2006; Matsunaga et al. 2004).

On the basis of a possible summative effect of time, a collateral interest of our study was to test whether or not anodal tDCS affects tactile performance in different epochs of stimulation. To this aim, we fractionated the $15 \mathrm{~min}$ of the online stimulation in three separate time windows: from 0 to 5 , from 6 to 10 , and from 11 to $15 \mathrm{~min}$. 
Table 3 Methodological characteristics and main results of the three experiments

\begin{tabular}{llll}
\hline & $\begin{array}{l}\text { Experiment 1 } \\
\text { (manual stimulation) }\end{array}$ & $\begin{array}{l}\text { Experiment 2 } \\
\text { (computerized stimulation) }\end{array}$ & $\begin{array}{l}\text { Experiment 3 } \\
\text { (computerized stimulation) }\end{array}$ \\
\hline Montage & Bicephalic & Monocephalic & Monocephalic \\
Task & Off-line & Online & Online \\
tDCS stimulation side & Right & Right-Left & Right \\
Tactile stimulation & Contralateral & Ipsilateral & Contralateral-Ipsilateral \\
Effect on accuracy & Yes & No & Yes (contra) \\
& & No (ipsi) \\
Effect on vRTs & Yes & Yes (only right) & Yes \\
\hline
\end{tabular}

We then performed ANOVA for repeated measures on accuracy and on vRTs from data of Experiments 2 and 3. Analyses showed no trend. Neither accuracy nor vRT significantly changed between epochs.

According to our knowledge, no systematic studies have focused on the duration of the tDCS effect over time in the posterior parietal areas, and indeed, this could be very important for those who use tDCS to rehabilitate neuropsychological patients.

\section{General discussion}

The main results can be summarized as follows. In the first study, we demonstrated the efficacy of tDCS in modulating subjects' performance in a distance discrimination task. This initial result seems to be of particular interest, since no previous studies have ever focused on the effectiveness of tDCS on higher order tactile functions such as those required in a tactile metric discrimination task.

Experiments 2 and 3 directly pointed at the functional properties of the angular gyrus (AG) of both hemispheres in dealing with tactile distance discrimination and, as a consequence, with the metric component of the mental body representations. Evidence from these two studies indicates that anodal stimulation on the right angular gyrus systematically reduces vocal reaction times (vRT), thus improving discrimination speed, independent of whether the stimuli are delivered to contralateral or ipsilateral body parts. However, accuracy improves only when the tactile stimuli are given on contralateral (left) limbs. Both speed and accuracy effects are totally absent when anodal stimulation is delivered to left AG. Given these results, it seems reasonable that right, but not left, angular gyrus is involved in the metric component of the MBR.

The involvement of parietal structures in the metric processing of MBR

In a previous fMRI study (Spitoni et al. 2010), we proposed that in human brain, we can distinguish two forms of touch perception: primary tactile sensation (such as an external object pressing on the skin) and secondary tactile perception that includes the metric/spatial information need to be rescale. We suggested that the extraction of the metric information from the skin surface involves additional computational processing stages over perceiving simple contact to the skin and that these rescaling processes are hosted by right $\mathrm{AG}$.

Evidence from the present study seems to confirm the key role played by the right $\mathrm{AG}$ in the rescaling processing of metrical features of MBR.

The involvement of parietal structures in the representations of the body has been widely investigated. According to Longo et al. (2010), parietal lobes play crucial roles in cognitive processes such as (1) the representation of the superficial body schema, (2) the localization of somatic stimuli on the body surface (Porro et al. 2007; Van Boven et al. 2005), (3) the perception of the current posture of the body (Pellijeff et al. 2006; Wolpert et al. 1998; Fasold et al. 2008), and (4) the structural/topological knowledge of one's own body (Felician et al. 2004; Corradi-Dell'Acqua et al. 2008). Specifically, the posterior parietal cortex (PPC) is thought to integrate different classes of sensory information (e.g., somatosensory, auditory, and visual) to generate multiple representations of space. Through combining these spatial representations, we can produce a variety of actions such as saccadic eye movements and reaching or grasping movements of the upper limb. Moreover, a recent TMS study by Azañón et al. (2010) provided clear evidence for a causal role of posterior parietal cortex (PPC) from a somatotopic to an external spatial frame of reference, suggesting a crucial role of this area in the remapping of touch.

Lesion studies in monkeys and in humans (Rushworth et al. 1997, 1998; Sirigu et al. 1995) suggest that cerebral areas within the PPC play an important role in maintaining an accurate and up-to-date representation of the current postural state of the body (the body schema). What seems of particular interest in this context is that the region selectively involved in the representations of the limbs postures is the superior parietal cortex (SPC), particularly the medial location (precuneus) (Pellijeff et al. 2006; Parkinson et al. 
2010). Moreover, a recent fMRI study with tactile stimulation over body surface (Huang et al. 2012) revealed somatotopic areas of the face and multiple body parts forming a higher level homunculus in the superior posterior parietal cortex.

In the present study, we stimulated the inferior parietal cortex (IPC), and we observed a modulation of the metrical component of MBR. Given these evidence, we can speculate that in order to build an accurate postural configuration, SPC may not need metrical rescaling and that this latter process could be selectively linked to IPC. The inferior and posterior parietal structures are also involved in localizing parts of one's own or others' bodies and in comparing the size of body parts. It should be noted that both the localization of parts and the comparison of distance over the body require activation of an internal body representation. In distance comparisons, however, the representation must include metric properties.

We are aware that tDCS has a low spatial resolution; that is why we believe that in order to claim to a clearer distinction between the functional roles of IPC and SPC, fMRI studies need to be implemented.

\section{Accuracy and speed in the metrical processing of MBR}

In our study, we focused on accuracy and speed, and we found an effect of tDCS on vRTs when electrical modulation was delivered to right $\mathrm{AG}$, and tactile stimulation was given to ipsilateral arms. Moreover, an effect of tDCS was seen on both vRTs and accuracy when anodal stimulation was delivered over the right AG, but tactile stimuli were given to the contralateral arms. These results seem to support an aspecific effect of tDCS on speed when tactile stimuli are delivered on the ipsilateral side of the electrical stimulation, together with a specific effect on accuracy when stimuli are given to the contralateral side. The reasons for such dissociation can only be hypothesized. On the one hand, we can assume the existence of two different mechanisms; the first could rely on the simple excitability changes in the somatosensory cortex contralateral to the side of tactile stimulation, whereas the observed vRTs reduction (on the body part ipsilateral to the stimulation) might simply reflect a more specific arousal effect of the anodal stimulation. What we know from previous studies is that in the tDCS setup, the current flows following routes that are influenced by the impedance of the tissue crossed by the current. This means that tissue impedance and cell orientation can strongly affect the flow of the current, resulting in different polarization patterns. Many studies in the literature assume that the maximum effect of tDCS occurs in areas perpendicular to the stimulating electrode and that cell polarization-depolarization is predominantly localized in these underlying brain structures
(Datta et al. 2010; Nitsche and Paulus 2011). But it has also been documented that a variable amount of current can also spread to contiguous areas (Datta et al. 2009, 2010; Sadleir et al. 2010). This latter evidence could partially give reason for the improvement of accuracy after contralateral stimulation; from this perspective, we can consider the possibility that an amount of current under right AG may spread to close somatosensory areas resulting in a known effect of improvement of tactile acuity of the contralateral side of stimulation (Ragert et al. 2008; Song et al. 2011).

\section{Methodological comments}

In our study, we used both mono-and bicephalic montages to test whether electrode disposition influenced performance on the tactile task. We found that anodal stimulation provided significant results in various conditions, that is, when the electrode montage was bicephalic or when the anodal electrode was placed on the brain and the cathode on the right shoulder.

Moreover, results did not change when tDCS was applied before the tactile discrimination task or during the stimulation. At variance with studies that measured other cognitive tasks (Andrews et al. 2011; Cohen Kadosh et al. 2010; Ross et al. 2010; Cerruti and Schlaug 2009), the effect was significant in terms of speeding responses and improving accuracy.

A limit of our experimental paradigm is the small amount of different distances on the skin that it was possible to stimulate through the mechanical devices used for Experiments 2 and 3. The solenoid device allowed for 8 miniature magnets only ( 4 on the thigh -4 on the forearm). With just 4 stimuli, we obtained a maximum of 3 sizes, and thus just 2 distance differences, that we labeled as "easy" and "difficult," respectively. This technical limitation prevented us to fit psychophysical functions to the raw data and analyze them in terms of parameters such as bias and precision, as we did in our previous study (Spitoni et al. 2010). Such analyses may bring to a clearer explanation on the nature of the improvement in accuracy found after tDCS.

To sum up, the present data suggest that anodal tDCS is effective in modifying the metric component of the MBR in a tactile discrimination tasks. Monocephalic montage of the electrodes and online stimulation produce consistent results, and in the specific task, these modifications have to be tested on parts of the body contralateral to the cortical stimulation.

Open Access This article is distributed under the terms of the Creative Commons Attribution License which permits any use, distribution, and reproduction in any medium, provided the original author(s) and the source are credited. 


\section{References}

Andrews SC, Hoy KE, Enticott PG, Daskalakis ZJ, Fitzgerald PB (2011) Improving working memory: the effect of combining cognitive activity and anodal transcranial direct current stimulation to the left dorsolateral prefrontal cortex. Brain Stimul 4:84-89

Azañón E, Longo MR, Soto-Faraco S, Haggard P (2010) The posterior parietal cortex remaps touch into external space. Curr Biol 27:1304-1309

Brunoni AR, Nitsche MA, Bolognini N, Bikson M, Wagner T, Merabet L, Edwards DJ, Valero-Cabre A, Rotenberg A, Pascual-Leone A, Ferrucci R, Priori A, Boggio PS, Fregni F (2012) Clinical research with transcranial direct current stimulation (tDCS): challenges and future directions. Brain Stimul 5:175-195

Cardini F, Longo MR, Driver J, Haggard P (2012) Rapid enhancement of touch from non-informative vision of the hand. Neuropsychologia 50:1954-1960

Cerruti C, Schlaug G (2009) Anodal transcranial direct current stimulation of the prefrontal cortex enhances complex verbal associative thought. J Cogn Neurosci 21:1980-1987

Cohen Kadosh R, Soskic S, Iuculano T, Kanai R, Walsh V (2010) Modulating neuronal activity produces specific and long-lasting changes in numerical competence. Curr Biol 23:2016-2020

Corradi-Dell' Acqua C, Hesse MD, Rumiati RI, Fink GR (2008) Where is a nose with respect to a foot? The left posterior parietal cortex processes spatial relationships among body parts. Cereb Cortex 18:2879-2890

Datta A, Bansal V, Diaz J, Patel J, Reato D, Bikson M (2009) Gyriprecise head model of transcranial direct current stimulation: improved spatial focality using a ring electrode versus conventional rectangular pad. Brain Stimul 2:201-207

Datta A, Bikson M, Fregni F (2010) Transcranial direct current stimulation in patients with skull defects and skull plates: high-resolution computational FEM study of factors altering cortical current flow. Neuroimage 52:1268-1278

de Vignemont F, Ehrsson HH, Haggard P (2005) Bodily illusions modulate tactile perception. Curr Biol 26:1286-1290

Dieckhöfer A, Waberski TD, Nitsche M, Paulus W, Buchner H, Gobbelé R (2006) Transcranial direct current stimulation applied over the somatosensory cortex - differential effect on low and high frequency SEPs. Clin Neurophysiol 117:2221-2227

Dijkerman HC, de Haan EH (2007) Somatosensory processes subserving perception and action. Behav Brain Science 30:189-201

Fasold O, Heinau J, Trenner MU, Villringer A, Wenzel R (2008) Proprioceptive head posture-related processing in human polysensory cortical areas. Neuroimage 15:1232-1242

Felician $\mathrm{O}$, Romaiguère $\mathrm{P}$, Anton JL, Nazarian $\mathrm{B}$, Roth $\mathrm{M}$, Poncet $\mathrm{M}$, Roll JP (2004) The role of human left superior parietal lobule in body part localization. Ann Neurol 55:749-751

Fuggetta G, Pavone EF, Walsh V, Kiss M, Eimer M (2006) Corticocortical interactions in spatial attention: a combined ERP/TMS study. J Neurophysiol 95:3277-3280

Huang RS, Chen CF, Tran AT, Holstein KL, Sereno MI (2012) Mapping multisensory parietal face and body areas in humans. Proc Natl Acad Sci USA 109(44):18114-18119

Hummel F, Celnik P, Giraux P, Floel A, Wu WH, Gerloff C, Cohen LG (2005) Effects of non-invasive cortical stimulation on skilled motor function in chronic stroke. Brain 128:490-499

Longo MR, Cardozo S, Haggard P (2008) Visual enhancement of touch and the bodily self. Conscious Cogn 17:1181-1191

Longo MR, Azañón E, Haggard P (2010) More than skin deep: body representation beyond primary somatosensory cortex. Neuropsychologia 48:655-668
Matsunaga K, Nitsche MA, Tsuji S, Rothwell JC (2004) Effect of transcranial DC sensorimotor cortex stimulation on somatosensory evoked potentials in humans. Clin Neurophysiol 115:456-460

Moyer RS, Bayer RH (1976) Mental comparison and the symbolic distance effect. Cogn Psychol 8:228-246

Nitsche MA, Paulus W (2011) Transcranial direct current stimulation-update 2011. Restor Neurol Neurosci 29:463-492

Oldfield RC (1971) The assessment and analysis of handedness: the Edinburgh inventory. Neuropsychologia 9:97-113

Parkinson A, Condon L, Jackson SR (2010) Parietal cortex coding of limb posture: in search of the body-schema. Neuropsychologia 48:3228-3234

Pellijeff A, Bonilha L, Morgan PS, McKenzie K, Jackson SR (2006) Parietal updating of limb posture: an event-related fMRI study. Neuropsychologia 44:2685-2690

Porro CA, Martinig M, Facchin P, Maieron M, Jones AK, Fadiga L (2007) Parietal cortex involvement in the localization of tactile and noxious mechanical stimuli: a transcranial magnetic stimulation study. Behav Brain Res 28:183-189

Ragert P, Vandermeeren Y, Camus M, Cohen LG (2008) Improvement of spatial tactile acuity by transcranial direct current stimulation. Clin Neurophysiol 119:805-811

Ross LA, McCoy D, Wolk DA, Coslett HB, Olson IR (2010) Improved proper name recall by electrical stimulation of the anterior temporal lobes. Neuropsychologia 48:3671-3674

Rushworth MFS, Nixon PD, Passingham RE (1997) Parietal cortex and movement. 1. Movement selection and reaching. Exp Brain Res 117:292-310

Rushworth MFS, Johansen-Berg H, Young SA (1998) Parietal cortex and spatial-postural transformation during arm movements. J Neurophysiol 79:478-482

Sadleir RJ, Vannorsdall TD, Schretlen DJ, Gordon B (2010) Transcranial direct current stimulation (tDCS) in a realistic head model. Neuroimage 51:1310-1318

Salmaso D, Longoni AM (1985) Problems in the assessment of hand preference. Cortex 21:533-554

Serino A, Haggard P (2010) Touch and the body. Neurosci Biobehav Rev 34:224-236

Sirigu A, Cohen L, Duhamel JR, Pillon B, Dubois B, Agid Y (1995) A selective impairment of hand posture for object utilization in apraxia. Cortex 31:41-56

Song S, Sandrini M, Cohen LG (2011) Modifying somatosensory processing with non-invasive brain stimulation. Restor Neurol Neurosci 29:427-437

Spitoni GF, Galati G, Antonucci G, Haggard P, Pizzamiglio L (2010) Two forms of touch perception in the human brain. Exp Brain Res 207:185-195

Taylor-Clarke M, Jacobsen P, Haggard P (2004) Keeping the world a constant size: object constancy in human touch. Nat Neurosci 7:219-220

Tsakiris M, Costantini M, Haggard P (2008) The role of the right temporo-parietal junction in maintaining a coherent sense of one's body. Neuropsychologia 46:3014-3018

Vallar G, Bolognini N (2011) Behavioural facilitation following brain stimulation: implications for neurorehabilitation. Neuropsychol Rehabil 21:618-649

Van Boven RW, Ingeholm JE, Beauchamp MS, Bikle PC, Ungerleider LG (2005) Tactile form and location processing in the human brain. Proc Natl Acad Sci USA 30:12601-12605

Wolpert DM, Goodbody SJ, Husain M (1998) Maintaining internal representations: the role of the human superior parietal lobe. Nat Neurosci 1:529-533 